\title{
Corrosión por picaduras del aluminio y de la aleación Al-6201 en soluciones de $\mathrm{NaCl}^{(\bullet)}$
}

\author{
R. Vera ${ }^{(*)}$, R. Schrebler ${ }^{(*)}$, G. Layana $(*)$, F. Orellana $(*)$ y A. Olguín $\left(^{* *}\right)$
}

\begin{abstract}
Resumen Gran cantidad de estudios realizados sobre el comportamiento del aluminio y sus aleaciones han demostrado su excelente resistencia a diversos medios corrosivos. Sin embargo, en las líneas de transmisión de energía eléctrica de aluminio o de la aleación comercial Al-6201, instaladas en las costas de Valparaíso, Chile, se detectaron fallos del material, consistentes en picaduras profundas y roturas. En este estudio se evalúa la corrosión por picaduras sufrida por el aluminio y la aleación $\mathrm{Al}$ 6201 , utilizando soluciones de diferentes concentraciones de $\mathrm{NaCl}$ que es el principal agente agresivo en ambientes marinos. Se determinaron los potenciales de corrosión $\left(E_{\mathrm{c}}\right)$, de repasivación $\left(E_{\mathrm{r}}\right)$ y de picado $\left(E_{\mathrm{p}}\right)$, mediante el empleo de curvas de polarización potenciodinámicas y técnica de raspado. La morfología del ataque se determinó mediante microscopía electrónica de barrido. Los resultados mostraron un comportamiento similar entre el aluminio y la aleación 6201. También se observó que el aumento en la concentración de cloruro conlleva una disminución en los potenciales de corrosión, de picaduras y de repasivación en ambos materiales.
\end{abstract}

Palabras clave: Aluminio. Aleación de aluminio. Corrosión por picaduras. Potenciales de corrosión. Picaduras y repasivación.

\section{Pitting by corrosion in aluminum and Al-6201 alloy}

\begin{abstract}
The susceptibility of pure aluminum and aluminum 6201 alloy to pitting was investigated in sodium chloride solutions through determination of the corrosion, repassivation and pitting potentials. Potentiodynamic polarization including scratching techniques were employed being also determined the type and relative amount of corrosion damage to the metals. The morphology of the attack was determined using scanning electron microscopy (SEM). The results showed a similar performance for aluminum 6201 alloy and aluminum. It was also observed that an increase in chloride concentration resulted in a decrease in the corrosion, pitting and repassivation potentials of both materials.
\end{abstract}

Keywords: Aluminum. Aluminum alloy. Pitting corrosion. Corrosion. Pitting and repassivation potentials.

\section{INTRODUCCIÓN}

Entre los metales utilizados en la actualidad en la construcción de líneas de transmisión eléctricas de alta tensión y redes de distribución, uno de los más resistentes a la corrosión atmosférica es el aluminio. Durante cientos de años ha mostrado ser más resistente que el acero y por décadas mejor que el zinc, especialmente en atmósferas urbanas.

(•) Trabajo recibido el día 17 de septiembre de 1997.

(*) Instituto de Química.

(**) Instituto de Física. Universidad Católica de Valparaíso. Avda. Brasil 2950, Casilla 4059. Valparaíso (Chile).
Entre sus principales propiedades merecen resaltarse su bajo peso específico, excelente resistencia a la corrosión y buena conductividad eléctrica. No obstante, el uso del aluminio puro es muy limitado por su baja resistencia mecánica. Esto ha originado el desarrollo de una gran variedad de aleaciones, tal como la aleación Al-6201 (Al-Si-Mg), usada especialmente para conductores eléctricos por presentar baja densidad, alta relación resistencia mecánica/peso y gran conductividad térmica y eléctrica (1-3).

La película de óxido formada sobre su superficie permite que con el tiempo su velocidad de corrosión disminuya, incluso en atmósferas industriales 
muy contaminadas (4). Sin embargo, cuando el aluminio se encuentra en contacto con otros metales, especialmente en atmósfera marina y en una menor área expuesta, puede sufrir corrosión por picaduras y galvánica, produciendo fallos prematuros, aun en aleaciones de aluminio con buenas propiedades mecánicas (5-7).

El comportamiento presentado por el aluminio y la aleación Al-6201 en $\mathrm{NaCl}$ a circuito abierto corresponde al de un metal en cuya superficie se forma y se estabiliza una película protectora. La causa fundamental de la corrosión por picaduras se atribuye a la rotura localizada de la película pasiva formada, lo que produce una disolución del material. Por esto, la resistencia a la corrosión por picaduras estaría determinada por la estabilidad electroquímica de la película y por su capacidad para regenerarse en el medio agresivo.

Estudios realizados para examinar la influencia de algunos parámetros atmosféricos sobre la formación y crecimiento de picaduras, durante la corrosión del aluminio, han demostrado que la densidad de picaduras aumenta linealmente con el tiempo de exposición, especialmente en atmósferas contaminadas con sustancias que contienen azufre y cloruros (8). Por otra parte, ensayos de corrosión realizados en Japón sobre alambres de aluminio usados como conductores evidenciaron un gran efecto acelerador del ataque y de la extensión del material dañado por acción de las lluvias y radiación solar (9). Asimismo, en estudios realizados por McIntyre (10) sobre la corrosión de aleaciones de aluminio de las series 6000 y 7000, expuestas a una solución de agua de mar artificial que contiene iones nitrato a diferentes temperaturas, se puso de manifiesto la existencia de un sinergismo entre los iones nitrato y cloruro, con aceleración de la corrosión intergranular de estas aleaciones.

Es sabido que en las redes eléctricas de las costas de Valparaíso (Chile) se han utilizado indistintamente aluminio y la aleación Al-6201, pero se ha detectado en la actualidad un aumento en la corrosión por picaduras de estos materiales, tal vez debido al incremento de contaminantes industriales, que potenciarían el efecto corrosivo del ambiente marino, como también por efecto de pares galvánicos producto de uniones de aluminio con los antiguos tendidos de cobre. Por lo anterior, en el presente trabajo, se realizaron estudios comparativos del comportamiento frente a la corrosión por picaduras, entre aluminio puro y la aleación Al-6201, para poder sugerir el uso preferencial de uno de estos materiales, en atmósfera marina, como conductor.

\section{PARTE EXPERIMENTAL}

Los ensayos se realizaron con muestras de aluminio 99,0 \% de pureza y de la aleación Al-6201 $(97,62 \% \mathrm{Al}, 0,7 \%$ Si y $0,9 \% \mathrm{Mg})$. La condición inicial del aluminio y de la aleación Al-6201 era material trefilado de diámetros $2,25 \mathrm{~mm}$ y $3,0 \mathrm{~mm}$, respectivamente.

Los ensayos electroquímicos fueron realizados empleando soluciones de $\mathrm{xM}$ de $\mathrm{NaCl}(0,01 \leq x \leq$ $1,0), \mathrm{pH}$ entre 5,5 y 6,0 , desaireadas con nitrógeno en una celda convencional de doble pared por la cual circulaba agua a $20^{\circ} \mathrm{C}$ proveniente de un termostato. El potencial de corrosión se obtuvo como el potencial a circuito abierto que adquiere el sistema al cabo de $120 \mathrm{~min}$, tiempo suficiente para alcanzar un valor estable. El potencial de corrosión por picaduras se determinó utilizando la técnica de raspado (11) y el de repasivación mediante el trazado de curvas de polarización en direcciones anódica y catódica (12).

Las medidas se obtuvieron empleando un potenciostato, un registrador y un generador con una velocidad de barrido de $1 \mathrm{mV} / \mathrm{s}$. Como contraelectrodo se usó un alambre de platino de gran área, y como electrodo de referencia uno de calomelanos saturado. El área expuesta de las probetas fue de 1 $\mathrm{cm}^{2}$ y se pulieron con papel de $\mathrm{SiC}$ granulometría 600 , lavadas con agua destilada y secadas con aire caliente. Con esta condición superficial previa del electrodo se logró una satisfactoria reproducibilidad en los resultados. Al término de cada medida se determinó la morfología de ataque del material mediante microscopía electrónica de barrido.

\section{RESULTADOS Y DISCUSIÓN}

En la tabla I se indican los potenciales de corrosión $\left(E_{\mathrm{c}}\right)$, de repasivación $\left(E_{\mathrm{r}}\right)$ y de picaduras $\left(E_{\mathrm{p}}\right)$, en una solución de $\mathrm{NaCl} 1 \mathrm{M}$, para aluminio y para la aleación A1-6201. En dicha tabla se observa que para ambos materiales, los tres parámetros considerados presentan valores similares.

Según Rosales et al. (13), la diferencia entre $E_{\mathrm{p}}$ y $E_{\mathrm{c}}$, provee una mejor información acerca de la medida de la susceptibilidad de un metal a la corrosión por picaduras, en un determinado medio. De acuerdo con los valores reportados en la tabla I no se aprecia una variación entre las diferencias de los potenciales mencionados, lo que indica un comportamiento similar en los dos materiales.

Considerando que la diferencia entre $E_{\mathrm{p}}$ y $E_{\mathrm{r}}$ proporciona información respecto de la susceptibilidad del material a la corrosión por resquicios, en la figura 1 se muestran las curvas de histérisis para aluminio y para la aleación Al-6201 en $\mathrm{NaCl} 1 \mathrm{M}$. 
TABLA I.- Potencial de corrosión, $E_{\mathrm{c}}$, potencial de picadura $E_{\mathrm{p}}$, y potencial de repasivación, $E_{\mathrm{r}}$, en $\mathrm{NaCl} 1 \mathrm{M}$, para aluminio y la aleación Al-6201

TABLE I.-Corrosion potential, $\mathrm{E}_{\mathrm{c}}$, pitting potentiaal, $\mathrm{E}_{\mathrm{p}}$ and repassivation potential, $\mathrm{E}_{\mathrm{r}}$, in $\mathrm{NaCl} 1 \mathrm{M}$, for aluminium and Al-6201 alloy

\begin{tabular}{|l|c|c|c|}
\hline Muestra & $E_{\mathrm{c}}, V$ & $E_{\mathrm{p}}, V$ & $E_{\mathrm{r}}, V$ \\
\hline Al & $-1,14$ & $-0,74$ & $-0,79$ \\
Al-6201 & $-1,12$ & $-0,73$ & $-0,80$ \\
\hline
\end{tabular}

Se observa un comportamiento similar para los dos materiales, ya que ambos poseen una zona de pasividad imperfecta semejante, lo cual sería un indicio de igual susceptibilidad a la corrosión en resquicios. Asimismo, las muestras estudiadas presentan una zona de pasividad similar, con una corriente del orden de $3,0 \times 10^{-6}\left(\mathrm{~A} \cdot \mathrm{cm}^{-2}\right)$.

En general, se observa que las diferencias entre $E_{\mathrm{p}}$ y $E_{\mathrm{r}}$ son pequeñas, de manera que, ante roturas eventuales de la película pasivante, es de esperar una fácil repasivación y una apreciable resistencia a la corrosión por picaduras en condiciones naturales de exposición de estos materiales. Sin embargo, en presencia de contaminantes industriales se han

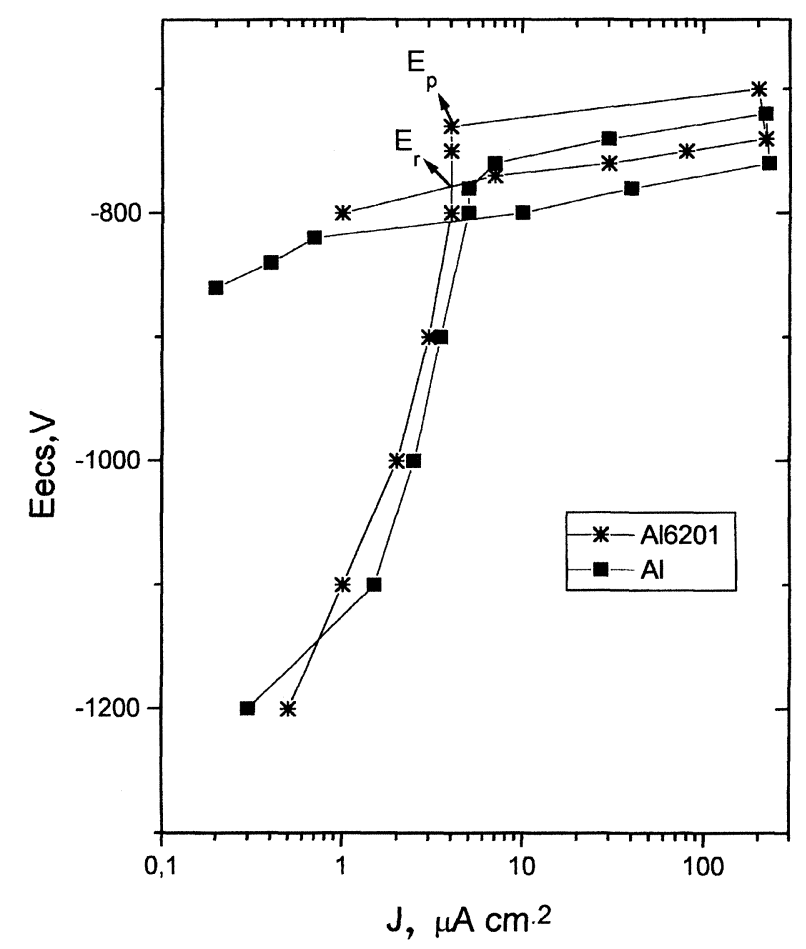

Fig. 1.- Curvas de polarización anódica para aluminio y la aleación Al-6201 en $\mathrm{NaCl} 1 \mathrm{M}$.

FIG. 1.- Anodic polarization curves of aluminium and Al-6201 alloy in $\mathrm{NaCl} 1 \mathrm{M}$. detectado fallos prematuros que podrían atribuirse a un efecto sinérgico del $\mathrm{SO}_{2}$ (14).

La figura 2 muestra el potencial de picaduras $\left(E_{\mathrm{p}}\right)$ en función de la concentración de ion agresivo, para aluminio y para la aleación Al-6201. En concordancia con los valores presentados en la tabla I, se observa que los potenciales de picaduras para ambos materiales se desplazan hacia valores más positivos con la disminución en la concentración de $\mathrm{NaCl}$ sentando una dependencia lineal con $p \mathrm{Cl}^{-}$.

La dependencia del potencial de picaduras con la concentración de cloruro se expresa en las ecuaciones [1a] para el aluminio y [1b] para la aleación.

$$
\begin{aligned}
& E_{\mathrm{p}}=-0,739+0,045 p \mathrm{Cl}^{-} \\
& E_{\mathrm{p}}=-0,724+0,049 p \mathrm{Cl}^{-}
\end{aligned}
$$

De las ecuaciones [1a] y [1b] se puede inferir que el comportamiento del potencial de picaduras en función de la concentración no difiere prácticamente de un material a otro. Al comparar los resultados proporcionados por otros autores (15-18) con los obtenidos en este trabajo, se tiene una coincidencia aceptable, tanto en el valor del $E_{\mathrm{p}}$ para soluciones $1 \mathrm{M}$ de cloruro, como en la pendiente de las rectas obtenidas.

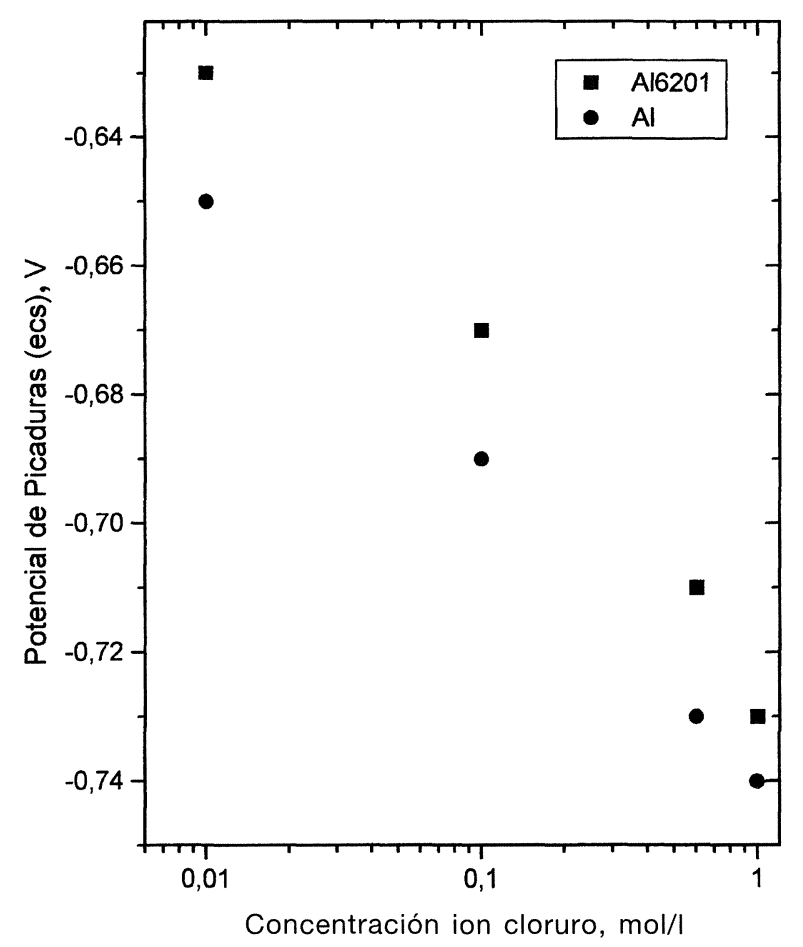

FIG. 2.- Potencial de picado en función de la concentración de ion cloruro para aluminio y la aleación Al-6201.

FIG. 2.- Pitting potential versus ion chloride concentration for aluminium and Al-6201 alloy. 
Con la finalidad de obtener mayor información del comportamiento de los metales en estudio, se realizaron ensayos corriente-tiempo a un potencial constante de $-800(\mathrm{mV})$, próximo al potencial de picadura y a un potencial de $30(\mathrm{mV})$ más positivo que $E_{\mathrm{p}}$. Estos resultados se muestran en la figura 3.

Como el primer potencial elegido para realizar el ensayo se encuentra en la zona de pasividad, la

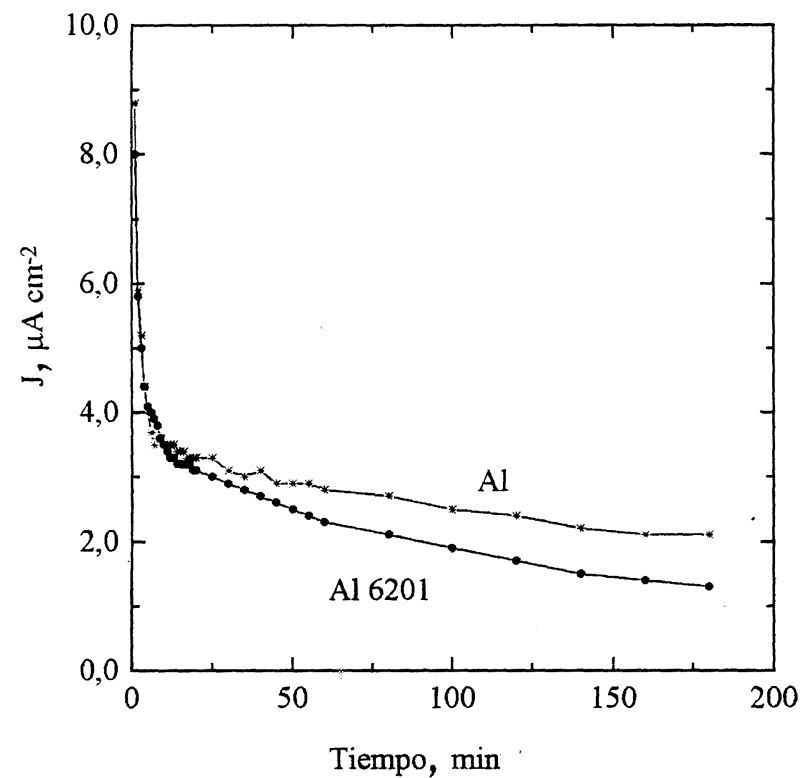

(a)

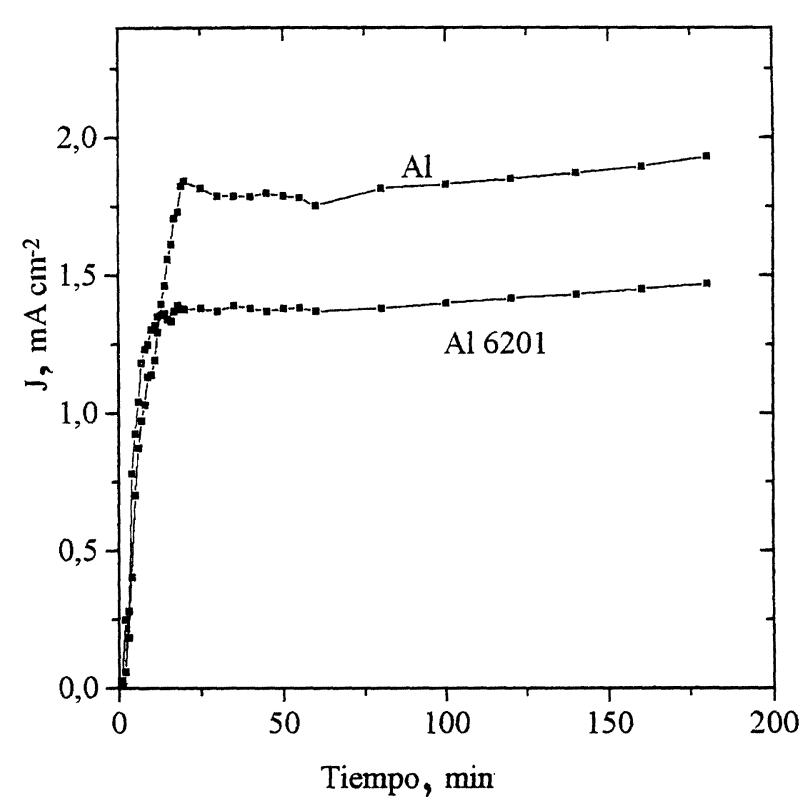

(b)

Fig. 3.- Densidad de corriente en función del tiempo para aluminio y la aleación Al-6201 en $\mathrm{NaCl} 1 \mathrm{M}$. a) $E=-800 \mathrm{mV}$ (ecs). b) $E=-700 \mathrm{mV}$ (ecs).

FIG. 3.- Current density versus time for aluminium and Al-6201 alloy in $\mathrm{NaCl} 1 \mathrm{M}$. a) $E=-800 \mathrm{mV}$ (sce). b) $E=-700 \mathrm{mV}$ (sce). corriente disminuye inicialmente debido a la formación de productos de corrosión pasivantes y se estabiliza rápidamente, mostrando un comportamiento similar para ambos materiales. El producto de corrosión determinado por difracción de rayos $\mathrm{X}$ para los dos materiales corresponde mayoritariamente a óxido de aluminio $\left(\mathrm{Al}_{2} \mathrm{O}_{3}\right)$ impurificado con $\mathrm{NaCl}$.

En las figuras $4 \mathrm{a}$ y $4 \mathrm{~b}$ se muestran las micrografías de la superficie del aluminio y de la aleación Al-6201, respectivamente. Las figuras 5 a y 5 b corresponden a las micrografías de un corte del aluminio y de la aleación Al-6201, respectivamente; en ellas se presenta la morfología de estos productos de corrosión. En las micrografías de la superficie se observa que el producto de corrosión generado sobre la aleación Al-6201 posee, aparentemente, mayor cristalinidad y mayor tamaño de
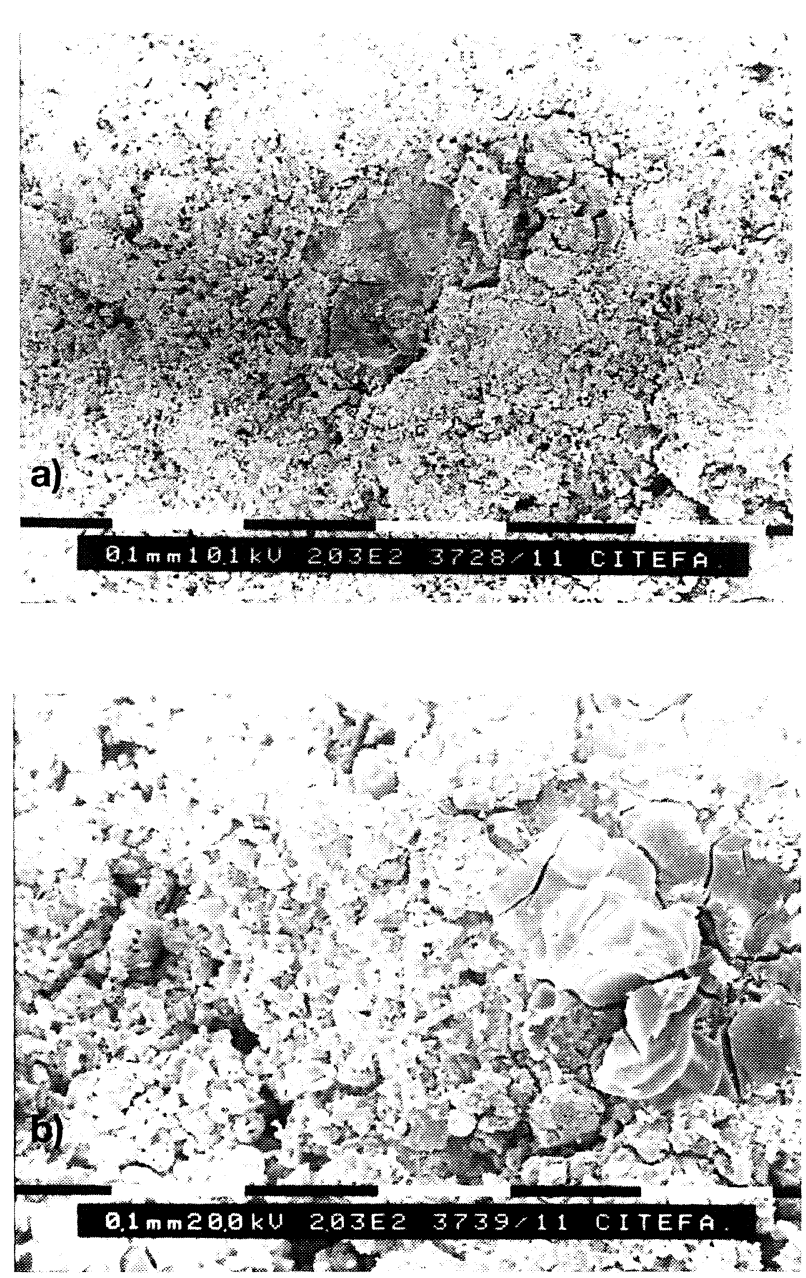

FIG. 4.- Micrografías de la superficie del material atacado en solución $1 \mathrm{M} \mathrm{NaCl}, \mathrm{a}-800 \mathrm{mV}$ (ecs). $\times 200$. a) Aluminio. b) Al-6201.

Fig. 4.- In plant micrographies of samples attacked in $1 \mathrm{M} \mathrm{NaCl}$ solution at $-800 \mathrm{mV}$ (sce). 200x. a) Aluminium. b) Al-6201. 

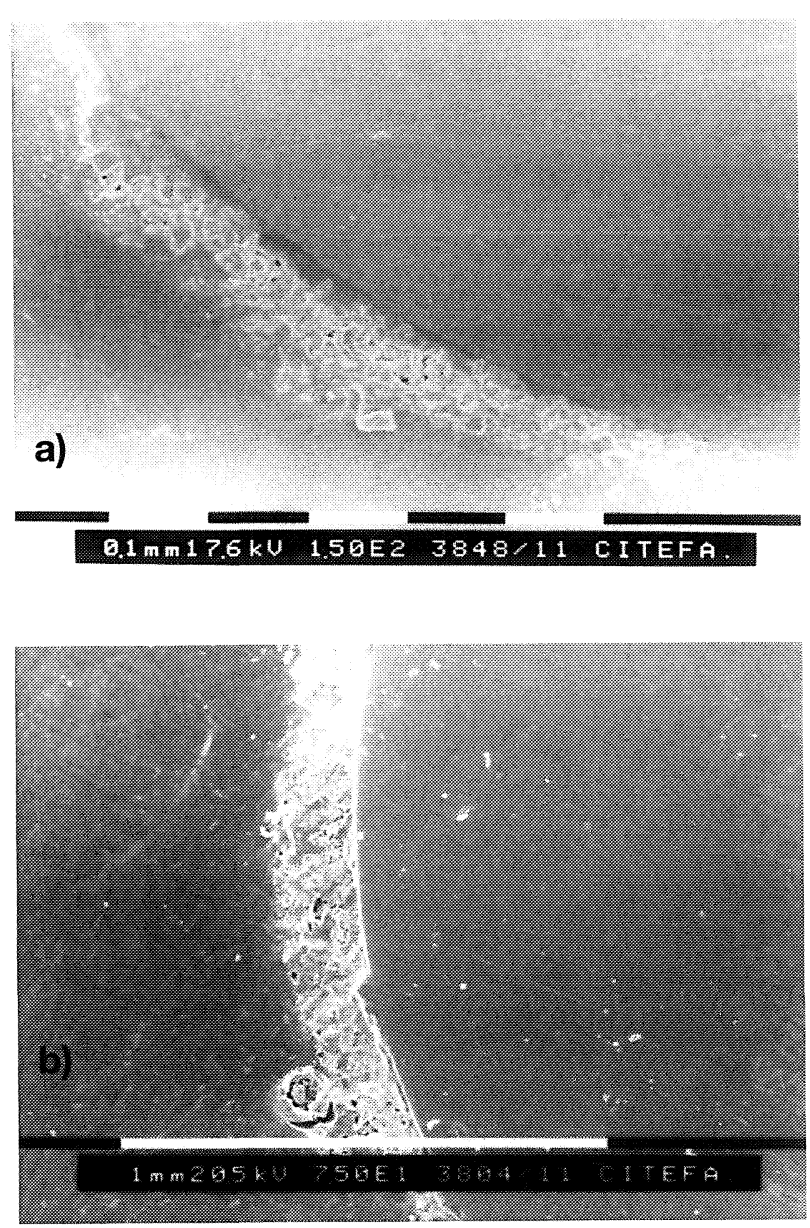

FiG. 5.- Micrografías de un corte transversal del material atacado en solución $1 \mathrm{M} \mathrm{NaCl}, \mathrm{a}-800 \mathrm{mV}$ (ecs). a) Aluminio. $\times 150$. b) Al-6201. $\times 75$.

FIG. 5.- Micrograph of the cross section of samples attacked in $1 \mathrm{M} \mathrm{NaCl}$ solution at $-800 \mathrm{mV}$ (sce). a) Aluminium. 150×. b) Al-6201 75×.

partículas, causados tal vez por la diferencia en la microestructura de los materiales en estudio. En las micrografías del corte (Figs. 5a y 5b) se muestra una mayor homogeneidad en el espesor de los productos de corrosión en la aleación.

Cuando la polarización se realiza a un potencial más positivo que $E_{\mathrm{p}}$ se observa un rápido aumento de corriente (Fig. 3), la cual se estabiliza a valores elevados, en concordancia con el ataque localizado determinado mediante técnicas de microscopía electrónica de barrido, como se observa en las micrografías del corte que se presentan en las figuras 6a (aluminio) y 6b (Al-6201). La morfología del ataque es similar para ambos materiales, lo que concuerda con lo citado por otros autores (10 y 19), ya que la aleación Al-6201 posee una relación silicio/magneso balanceada que le permite formar un compuesto estequiométrico de $\mathrm{Mg}_{2} \mathrm{Si}$, sin quedar exceso de silicio, evitando así un ataque por corrosión intergranular. a)

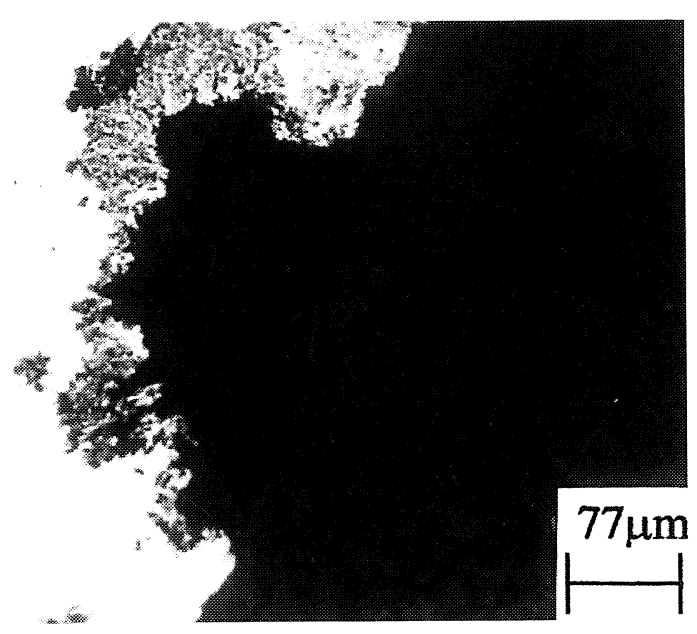

b)

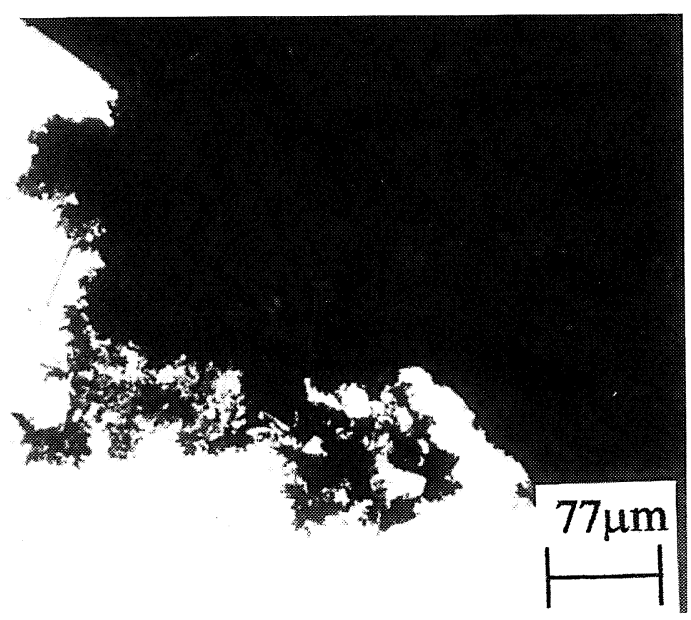

FIG. 6.- Micrografías de un corte transversal del material atacado en solución $1 \mathrm{M} \mathrm{NaCl} \mathrm{a-700} \mathrm{mV}$ (ecs). $\times 130$. a) Aluminio. b) Al-6201.

FIG. 6.- Micrograph of the cross section of samples attacked in $1 \mathrm{M} \mathrm{NaCl}$ solution at $-700 \mathrm{mV}$ (sce). $130 \times$ a) Aluminium b) Al-6201.

\section{CONCLUSIONES}

De la investigación realizada, se puede concluir que entre el aluminio y la aleación Al-6201 no existe una diferencia en el comportamiento frente a la corrosión por picaduras en medios con cloruros que justifique aconsejar el uso de uno de los materiales frente al otro. Por esto, si se decide usar aluminio en líneas de transmisión de energía eléctrica en atmósferas marinas, se puede utilizar indistintamente aluminio puro o la aleación Al-6201.

\section{Agradecimiento}

Los autores desean manifestar su agradecimiento a la Dirección General de Investigación y Postgrado de la Universidad Católica de Valparaíso, Proyecto 125.766; a la empresa Chilquinta Energía 
y a la Dra. Blanca Rosales por su colaboración en la obtención de las micrografías con MEB en el CITEFA (Argentina).

\section{REFERENCIAS}

(1) Silveira, V. Alguns tópicos referentes a aplicaçao do aluminio para conduçao elétrica, Centro de Pesquisas de Energia Elétrica, 1975.

(2) Meduna, E. Estudo de Linhas de Transmissao, VII Seminario Nacional de Produçao e Transmissao de Energia Elétrica, Brasilia (Brasil), 1983.

(3) Yanque, J. Corrosión de Conductores Eléctricos de Aluminio y sus Aleaciones Ligeras. Primer Seminario de Corrosión y Técnicas de Protección. Lima (Perú), 1985.

(4) Alcan Research and Development Limited. Corrosion considerations in overhead aluminum conductors. Canadá, 1986.

(5) Chen, G., GaO, M. y Wei, R. Corr. Sci., 52 (1), 1996: 815.

(6) Wu, T. y Wu, J. Corr. Sci., 51 (3), 1995: 185-190.

(7) Buorgum, A., Sigurdsson, H. y Nisancioglu, K. Corr. Sci., 51 (7), 1995: 544-557.
(8) Elola, S., Otero, T. y Porro, A., Corr. Eng., 48 (10), 1992: 854-863.

(9) YasuYuki, U. Study on Corrosion of Insulated Aluminum Wires due to Salt Contamination. Japan Iere Council, R8420, Japón, 1984

(10) McIntyre, F. y Dow, T. Corr. Sci., 48, 1992: 309-319.

(11) Pessall, N. y Liu, C. Electrochim. Acta, 16, 1971: 1987.

(12) Pourbaix, M. Atlas of Electrochemical Equilibria. Pergamon Press, Oxford, 1974.

(13) Ayllon, S. y Rosales, B.M., Corr. Sci., 50 (8), 1994: 571-575.

(14) Vera, R., Martinez, L., Layana, G, Orellana F. y Olguin, A. Corrosion in Electrical Power Lines in Coastal Areas of Valparaíso@, 2nd NACE Latin American Region Corrosion Congress, Rio de Janeiro (Brasil), 1996.

(15) Raspini, A., Corr. Sci., 49 (10), 1993: 821-828.

(16) Damborenea, J. y Conde, A. Br. Corros. J., 30 (4), 1995: 292-296.

(17) Staia, M., Puchi, E. y Vera, R. Acta Microsc., 1 (1), 1992: 29-42.

(18) Burleigh, T., Rennich, R. y Bovard, F., Corr. Sci, 49 (8), 1993: 683-685.

(19) Elola, A., Otero, T. y Porro, A., Corrosion, 48 (10), 1992: 854-863 